\title{
Interactive comment on "Snowfall versus sub-shelf melt: response of an idealized 3D ice-sheet-shelf system to mass redistribution" by Johannes Feldmann et al.
}

\section{Anonymous Referee \#1}

Received and published: 23 August 2018

The paper entitled "Snowfall versus sub-shelf melt: response of an idealized 3D icesheet-shelf system to mass redistribution" by Feldmann, Reese, Winkelmann and Levermann investigates the response of a model to an increase in surface snow accumulation balanced by an increase in basal melting under its floating part. The idea is that we expect Antarctica to receive more snowfall (mass gain) and at the same time experience enhanced ocean-induced basal melting (mass loss) and it is not clear how the overall volume of the ice sheet is going to change. Here, the authors use PISM and a setup derived from MISMIP+ to investigate this question. They start the model from a steady state and apply an increase in surface accumulation over grounded ice that exactly balances the increase in basal melt applied under floating ice, and the model

Printer-friendly version

Discussion paper 
runs forward until a new steady state is found. The authors find that, in all cases, the grounding line of the model does retreat, but the total volume of the system increases, leading to a net volume gain. Overall the paper is well written, easy to follow, but I have some important recommendations that should be addressed before the paper can be accepted for publication.

\section{General comments}

While I am sure that these conclusions are robust, I think that they may still be specific to this setup and may not be generalized to all systems. More specifically, I think the authors do a good job at testing the sensitivity of their conclusions to some parameters (such as ice softness or bed slopes), I think they are still missing an important parameter: basal friction.

Overall, I really struggled with is the lack of detail in the model setup. The authors do mention that they rely on the MISMIP+ experiment but do not provide any boundary conditions in the manuscript, which I think is absolutely necessary in any modeling paper. One key ingredient in the response of the model is basal friction and I could not figure out what is being used here. To be clear, I do believe that the grounding line would retreat moderately if there is a lot of basal friction, but I expect to see much more grounding line retreat for more "slippery" beds. In other words, the initial increase in basal melt is going to lead to "some" grounding line retreat, and if the ice is sliding more, the increase in ice velocity will extend far more upstream and the wave of thinning is therefore expected to propagate significantly upstream (all the way to the ice divide). The amplitude of thinning can potentially be stronger than the thickening due to a larger accumulation.

If I follow the MISMIP+ paper the authors refer to, participants are free to use any of the following laws:

Printer-friendly version

Discussion paper 
- a power law

- a modified power-law relation introduced by Tsai et al. (2015)

- a modified power-law relation introduced by Schoof (2005) and explored by Gagliardini et al. (2007) and Leguy et al. (2014)

Which one is used here? I expect the model to respond differently depending on the basal friction law used, but also the friction parameter, $\beta^{2}$.

I recommend dividing the friction parameter $\beta^{2}$ by 2, 10 or even 100 to see how this impacts the model.

It is more minor but I am not $100 \%$ convinced that significantly higher snowfall has been observed in Antarctica, when significantly stronger melt has been measured, especially close to the grounding lines. So, making the argument that the anomalies will "cancel out" might not necessarily be true. On this note, I found it hard to translate the total applied perturbation, provided in Gt/yr, into accumulation/melting rates in $\mathrm{m} / \mathrm{yr}$. It would be nice to have a rough idea of what these anomalies translate into, in terms of rates. Right now, I don't know if the perturbations are realistic or not. I expect, for example, the melt to change by several $10 \mathrm{~s}$ of $\mathrm{m} / \mathrm{yr}$, and the accumulation by a few $\mathrm{cm} / \mathrm{yr}$ at most. Is that what's used here?

\section{Minor comments}

There is not a lot of punctuation in the manuscript, which makes it hard to read at times. For example, there should be a comma before "which" (most of the time), or "where". This sentence for example is clearly missing commas: "... the highest basal melt rates of Antarctic ice shelves are observed close to the grounding line where the 
ice shelf is thickest which is typically ...", or "Using the model setup described above the simulations are initiated with a block ...", etc.

- p1 12: under future warming, which leads to ... (missing comma)

- p1 120: , which regulates (missing comma)

- p2 19: experiments, such backstress reduction (missing comma)

- p2 I25: the pattern of sub-ice-shelf melting, we carry out (missing comma)

- p3 11: I think the references are wrong: Morland 1987 is for SSA (you should also cite MacAyeal 1989) and Hutter 1983 is for SIA.

- p3 15: how is melt applied on partially floating cells? A lot of recent work has shown that this might have a strong impact for resolutions on the order of $1 \mathrm{~km}$ or more.

- p3 18 during ice-sheet spinput, the rate (missing comma)

- p3 I12:feeding a bay-shaped ice shelf, which (missing comma)

- p3 126: the model described above, the simulations ... (missing comma)

- p3 I31: molten $\rightarrow$ melted

- p4 122: This way, a set of ... (missing comma)

- p4 125: consider replacing · by $\times$

- p4 I29: a bay-shaped ice shelf, which (missing comma)

- p5 128: is characterized by grounding-line retreat and an increase in ice volume 
- p5 129: perturbation, here the ... (missing comma)

- p6 12: effecting $\rightarrow$ resulting in a smaller ...

- p7 123: In the following, we carry out (missing comma)

- p8 115: 10\% (no space between 10 and \%)

- p8 118: , which the lateral (missing comma)

- p8 I20: This fits out $\ldots \rightarrow$ This is consistent with $\ldots$

- p8 125: , we show that (missing comma)

- p9 123: reducing the concentration of sub-ice-shelf melting close to the grounding line?

- p10 19: Quantification

- p10 I11: in ice-shelf length, which ... (missing comma)

- p10 128: At the same time, out approach... (missing comma)

Interactive comment on The Cryosphere Discuss., https://doi.org/10.5194/tc-2018-109, 2018. 